\title{
The utility of the tumor markers CA15.3, CEA, CA-125 and CA19.9 in metastatic breast cancer
}

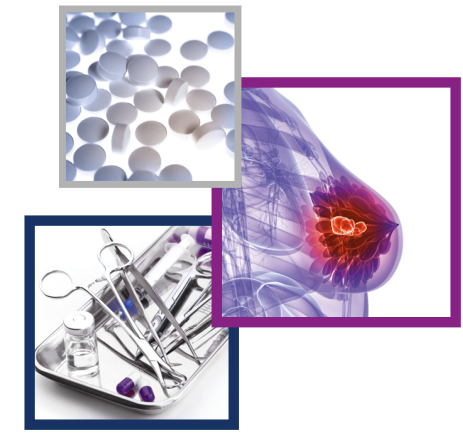

\author{
Gregory Gaughran*,1 (i), Neha Aggarwal ${ }^{1}$, Bruce Shadbolt ${ }^{2,3}$ \& Robin Stuart-Harris ${ }^{1,2}$ \\ 1 Department of Medical Oncology, The Canberra Hospital, Garran, ACT, 2605, Australia \\ ${ }^{2}$ ANU Medical School, Australian National University, Barry Drive, Acton, ACT, 2000, Australia \\ ${ }^{3}$ Centre for Health \& Medical Research ACT Health, Building 2-6, Level 3, Bowes Street, Garran, ACT, 2605, Australia \\ *Author for correspondence: gregorygaughran@gmail.com
}

Background: Cancer antigen 15.3 (CA15.3) is a commonly used tumor marker (TM) in metastatic breast cancer (MBC) but may not be raised. We assessed CA15.3, carcinoembryonic antigen (CEA), cancer antigen 125 (CA-125) and cancer antigen 19.9 in 193 MBC patients at diagnosis and follow-up. Materials \& methods: This TM panel was measured approximately 3 monthly. Median follow-up was 29.3 months. Results: At diagnosis, the following TMs were raised: CA15.3 63.2\%, CEA 37.3\%, CA-125 45.0\% and cancer antigen $19.917 .3 \%$. CA15.3 became raised later in 28/71. Raised TMs were less common in HER2+ tumors. CA-125 was frequently raised in triple negative tumors and was associated with pleural metastases. More raised TMs correlated with more sites of metastases and shorter survival. Conclusion: CEA and CA-125 showed benefit over CA15.3 alone in MBC and all three should be considered in MBC.

First draft submitted: 14 March 2020; Accepted for publication: 4 August 2020; Published online: 18 September 2020

Keywords: CA-125 • CA15.3 • CA19.9 • CEA • metastatic breast cancer • tumor markers

Although, there was a significant decrease in breast cancer mortality in Australia between 1990 and 2000 [1], we recently reported that $>12 \%$ of women treated for early breast cancer subsequently develop distant metastases [2]. Moreover, about $6 \%$ of women have metastatic breast cancer (MBC) at presentation [3]. Disease evaluation often includes a biopsy of a metastasis and assessment of tumor estrogen receptor (ER), progesterone receptor (PgR), tumor HER2 status and computerised tomography (CT) staging and a whole-body bone scan. Serum tumor markers (TMs) are commonly used and, in conjunction with imaging, provide a cost-effective way of supporting the diagnosis of $\mathrm{MBC}$ and also monitoring the response of the disease to therapy. The most commonly used TMs in MBC are: CA15.3, CEA and cancer antigen 27.29 (CA27.29) and all three are recommended for use in MBC by the American Society of Clinical Oncology (ASCO) [4].

In the USA, CA27.29 is used whereas in Australia CA15.3 is measured, however both have significant overlap and similar performance [5]. Both measure sequences of mucin that are secreted epitopes of MUC1, a protein derived from transcription of the $M U C 1$ gene, frequently overexpressed in a number of epithelial derived malignancies including breast cancer. MUC1 appears to have anti-adhesive properties, potentially facilitating metastatic spread [6]. In normal adults, CEA, an oncofetal protein, is present in low levels in mucosal tissue. The CEA family of glycoproteins show deregulated cell surface expression in malignancy, particularly in adenocarcinomas, with aberrations causing in vitro evidence of altered tissue architecture, adhesion as well as apoptotic evasion [7].

In MBC, CA15.3 has been reported to be raised in up to $80 \%$ of patients whereas CEA has only been reported to be raised in up to $65 \%$ of patients [8]. CA- 125 may be raised in about $35 \%$ of patients with MBC [9]. CA-125, also known as MUC16, serves as a lubricant and barrier in normal ocular, respiratory and female reproductive systems. CA-125 has also been shown to bind to mesothelin, a glycoprotein that lines peritoneal and pleural tissue which, in vitro, allows MUC16 to propagate in those coelomic tissues [10]. There are limited data for CA19.9 in MBC but it too may be raised [11]. However, measurement of CA19.9 has the confounder that $6-22 \%$ of the general population have an enzyme deficiency required to produce this antigen [12]. In MBC, CA15.3 is more sensitive 
than the other three markers, but there are some MBC patients that do not have a raised CA15.3. There are no guidelines to assist the clinician on what to do when CA15.3 is negative in a patient with MBC.

We have investigated the utility of CA15.3, CEA, CA-125 and CA19.9 in MBC. Our aims were to examine whether there was benefit in using these other TMs in addition to CA15.3, to examine the utility of continued measurement of CA15.3, CEA, CA-125 and CA19.9, when CA15.3 was not raised at diagnosis and to determine whether there are any correlations between raised $\mathrm{TM}(\mathrm{s})$ and sites of metastases, tumor subtype and survival.

\section{Materials \& methods}

A retrospective review was conducted of all newly diagnosed MBC patients presenting to two breast clinics at two hospitals in the Australian Capital Territory between 1 December 2002 and 30 June 2018 that had CA15.3, CEA, CA-125 and CA19.9 measured at diagnosis and in follow-up. Where possible, a biopsy of a metastasis was performed to confirm the diagnosis of MBC. Patients were required to have imaging evidence supportive of metastatic disease. Follow-up was closed at 31 May 2019.

Data collected were: patient age, patient gender, tumor histology, site(s) of metastases and date of metastatic disease, defined as the date of the biopsy or when MBC was diagnosed clinically if a biopsy was not available. The study was approved by the Australian Capital Territory Health Human Research Ethics Committee (reference number ETHLR.17.142). Excluded were patients without three or more baseline TMs within 4 weeks from diagnosis, those without all four TMs at 3 months from diagnosis, those without imaging at diagnosis and those with synchronous other malignancies.

Tumors were classified into three subtypes: hormone receptor positive (HR $+[\mathrm{ER}+$ and/or PgR-positive $])$, HER2-positive ([HER2+] HR-) and triple negative (TN [ER-, PgR-, HER2-]) as determined by the biopsy of a metastasis or the histopathology of the original tumor if a biopsy of a metastasis was not available.

All imaging reports and clinical information were reviewed to determine sites of metastases at diagnosis. Baseline CA15.3, CEA, CA-125 and CA19.9 at diagnosis were measured at one of two local laboratories and repeated at follow-up appointments. A raised TM was defined as any TM value $\geq 15 \%$ above the upper limit of normal. Imaging was routinely performed on all patients at approximately 3-monthly intervals or as clinically indicated. Follow-up was from the date of diagnosis of MBC to the date of the last TMs or the date of death. Sites of metastases were classed as: pleural and/or peritoneal, visceral (lung, liver), bone and nodal.

Descriptive analyses were employed as an overview of general findings, using binomial distributions associated with tumor subtype and sites of metastases. Generalized linear models were used to analyze the associations between the raised TMs and the tumor subtype, sites of metastases and survival. The type of generalized model used was determined by the outcome measure. These included logistic regression where the outcome was binary, multinomial regression where the outcome was categorical $(>2)$, Poisson regression where the outcome was a count and Cox regression for survival. Significant predictors for inclusion in the models were considered to have a p-value $<0.05$. Multivariable regression analyses were performed to adjust for patient age and to determine the associations between clinical indicators and survival, using a stepwise method. The clinical indicators were: individual raised TMs, number of raised TMs, sites of metastases and the combinations of raised TMs. Data were analyzed using the statistical package SPSS v.26.

\section{Results}

Of 202 potentially eligible subjects, nine were excluded: four because of no TM results within 1 month of diagnosis, two because of locoregional disease only and three because of a second malignancy, leaving 193 eligible subjects. Clinicopathological characteristics of the 193 subjects are shown in Table 1. Median follow-up was 29.3 months (range <1.0-146.9 months). 184/193 (95.3\%) had a biopsy of a metastasis; 129/193 (66.8\%) have died, but $54 / 193(28.0 \%)$ were alive at 31/05/2019. The survival of $10 / 193(5.2 \%)$ is unknown.

\section{Tumor markers at the diagnosis of metastatic disease}

As shown in Table 2, a total of $168(87.0 \%)$ had all four markers measured at diagnosis. In these, the following TMs were raised: one in $52(31.0 \%$, most commonly CA15.3), two in $49(29.2 \%)$, three in $22(13.1 \%)$ and four in 12 (7.1\%). A total of 33 of $189(17.5 \%)$ had no raised TM at diagnosis, but 156/189 (82.5\%) had at least one raised TM. CA15.3 was the most commonly raised TM at diagnosis (122/193, 63.2\%), followed by CA-125 (86/191, 45.0\%), CEA (72/193, 37.3\%) and, at last, CA19.9 (29/168, 17.3\%). 
Table 1. Clinicopathological characteristics of the patients.

\begin{tabular}{|c|c|c|}
\hline Patients & 193 & \\
\hline Gender & 192 females & 1 male \\
\hline Age & Mean 63.5 years & Range $30.8-90.7$ years \\
\hline \multicolumn{3}{|l|}{ Tumor } \\
\hline Biopsy at diagnosis (\%) & $184(95.3)$ & \\
\hline HR-positive (\%) & $131(67.9)$ & \\
\hline HER2-positive $^{\dagger}(\%)$ & $36(18.7)$ & \\
\hline TN $(\%)$ & $26(13.5)$ & \\
\hline Histology type (\%) & IDC 169 (87.6) & ILC 20 (10.4) \\
\hline \multicolumn{3}{|l|}{ Sites of metastases } \\
\hline Nodal (\%) & $86(44.6)$ & \\
\hline Visceral ${ }^{\ddagger}(\%)$ & $109(56.5)$ & \\
\hline Osseous (\%) & $123(63.7)$ & \\
\hline Pleural/peritoneal (\%) & $34(17.6)$ & \\
\hline \multicolumn{3}{|l|}{ Survival as at 31 May 2019} \\
\hline \multicolumn{3}{|l|}{$\begin{array}{l}\text { Study period } 01 \text { December 2002-30 } \\
\text { June } 2018\end{array}$} \\
\hline Alive (\%) & $54(28.0)$ & \\
\hline Deceased (\%) & $129(66.8)$ & \\
\hline Survival unknown (\%) & $10(5.2)$ & \\
\hline Mean survival patients alive & 13.5 years & Range 57 days -14.5 years \\
\hline Mean survival patients deceased & 4.0 years & Range 3 days -9.5 years \\
\hline \multicolumn{3}{|c|}{$\begin{array}{l}\text { †HER2-positive excludes those with hormone receptor positive status. } \\
\text { †Visceral: Lung, or liver or brain metastases. } \\
\text { HR-positive: Hormone receptor-positive; IDC: Infiltrating ductal carcinoma; ILC: Infiltrating lobular carcinoma; TN: Triple negative. }\end{array}$} \\
\hline
\end{tabular}

\begin{tabular}{|c|c|c|c|c|}
\hline At diagnosis & CA15.3 & CEA & CA-125 & CA19.9 \\
\hline Available for analysis (\%) & 193/193 (100.0) & $193 / 193(100.0)$ & $191 / 193(99.0)$ & $168 / 193(87.0)$ \\
\hline Raised at diagnosis (\%) & $122 / 193(63.2)$ & $72 / 193(37.3)$ & $86 / 191(45.0)$ & $29 / 168(17.3)$ \\
\hline No marker raised at diagnosis $(\%)$ & $33 / 168(19.6)$ & & & \\
\hline One marker raised $(\%)$ & $52 / 168(31.0)$ & & & \\
\hline Two markers raised $(\%)$ & $49 / 168(29.2)$ & & & \\
\hline Three markers raised $(\%)$ & $22 / 168(13.1)$ & & & \\
\hline Four markers raised $(\%)$ & $12 / 168(7.1)$ & & & \\
\hline \multicolumn{5}{|l|}{ CA15.3 not raised 71/193 (36.8\%) at diagnosis } \\
\hline Another marker raised $(\%)$ & & $13 / 71(18.3)$ & $23 / 71(32.4)$ & $6 / 66(9.1)$ \\
\hline Raised later (\%) & 28/71 (39.4) & $11 / 71(15.5)$ & $10 / 71(14.1)$ & $4 / 71(5.6)$ \\
\hline At least one non-CA15.3 marker raised (\%) & $34 / 67(50.1)$ & & & \\
\hline Total at least one marker raised (\%) & $156 / 189(82.5)$ & & & \\
\hline \multicolumn{5}{|l|}{ In follow-up } \\
\hline Total pts with CA15.3 ever raised (\%) & $150 / 193(77.7)$ & & & \\
\hline CA15.3 never raised $(\%)$ & $43 / 193(22.3)$ & & & \\
\hline No markers ever raised $(\%)$ & 19/193 (9.8) & & & \\
\hline All four markers raised $(\%)$ & $12 / 168(7.1)$ & & & \\
\hline
\end{tabular}

Tumor markers when CA15.3 was normal at diagnosis

As in Table 2, off the 71/193 (36.8\%) with a normal CA15.3 at diagnosis, 34/67 (50.1\%) had one or more TM raised: CEA in 13/71 (18.3\%), CA-125 in 23/71 (32.4\%) and CA19.9 in 6/66 (9.1\%). Therefore, in patients with a normal CA15.3 at diagnosis, CA-125 provided more patients with a raised TM than either CEA or CA19.9. 
Table 3. Raised tumor markers according to tumor subtype at diagnosis; analysis by stepwise logistic regression.

\begin{tabular}{|lllll|}
\hline Tumor & CA15.3 & CEA & CA-125 & CA19.9 \\
\hline HR $+(\%)$ & $96 / 131(73.3)^{\dagger}$ & $60 / 131(45.8)^{\ddagger}$ & $61 / 131(46.6)$ & $20 / 114(17.5)$ \\
\hline HER2+ (\%) & $15 / 36(41.7)$ & $8 / 36(22.2)$ & $8 / 35(22.9)$ & $2 / 28(7.1)$ \\
\hline TN (\%) & $11 / 26(42.3)$ & $4 / 26(15.4)$ & $18 / 26(69.2)^{\dagger}$ & $7 / 26(26.9)$ \\
\hline
\end{tabular}

$\dagger p<0.001$

$\ddagger p<0.002$.

HR+: Hormone receptor-positive; HER2+: HER2-positive; TN: Triple negative.

Table 4. Number of raised tumor markers by tumor subtype at diagnosis.

Number of raised markers (168) Tumor subtype

\begin{tabular}{|c|c|c|c|}
\hline & \\
\hline & $\mathrm{HR}+(114)$ & HER2+ (28) & TN (26) \\
\hline None (33) & $15 / 33(45.5 \%)$ & $12 / 33(36.4 \%)$ & $6 / 33(18.2 \%)$ \\
\hline One (52) & $34 / 52(65.4 \%))$ & $10 / 52(19.2 \%)$ & $8 / 52(15.4 \%)$ \\
\hline Two (49) & $37 / 49(75.5 \%)$ & $6 / 49(12.2 \%)$ & $6 / 49(12.2 \%)$ \\
\hline Three (22) & $18 / 22(81.8 \%)$ & $0 / 22$ & $4 / 22(18.2 \%)$ \\
\hline Four (12) & $10 / 12(83.3 \%)$ & $0 / 12$ & $2 / 12(16.7 \%)$ \\
\hline Average number of raised TMs & 1.8 & 0.8 & 1.5 \\
\hline
\end{tabular}

HR+: Hormone receptor-positive; HER2+: HER2-positive; TN: Triple negative; TM: Tumor marker.

CA15.3 became raised later in 28/71 (39.4\%) after a median of 23.4 months (range: 10 days-7.4 years). In addition, the following TMs, which had not been raised at diagnosis in this cohort, became raised during follow-up: CEA in $11 / 71$ (15.5\%), CA-125 in 10/71 (14.1\%) and CA19.9 in 4/71 (5.6\%). At the end of the study, the total number of patients with a raised CA15.3 was 150/193 (77.7\%). No TM was ever raised in 19/193 (9.8\%).

\section{Tumor markers \& tumor subtypes}

At diagnosis, the average number of raised TMs according to tumor subtype were: HR+ tumors 1.8 , TN tumors 1.5 and HER2+ tumors 0.8. Raised TMs occurred significantly more frequently in HR+ tumors (86.8\%) than TN $(76.9 \%)$ or HER2+ tumors $(57.1 \%, \mathrm{p}=0.02$, Chi-square test). HER2+ tumors were significantly less likely to have a raised TM than either HR+ $(\mathrm{p}<0.0001)$ or TN tumors $(\mathrm{p}=0.03$, Poisson regression, Bonferroni adjusted p-values), see Tables $3 \& 4$.

CA15.3 was the most frequently raised TM in all tumor subtypes, except for TN tumors where CA-125 was raised more frequently ( 42.3 vs $69.2 \%$, respectively). CA 15.3 was significantly more likely to be raised in HR+ $(73.3 \%)$ than either HER2+ $(41.7 \%, \mathrm{p}=0.001)$ or TN tumors $(42.3 \%, \mathrm{p}<0.0001$, logistic regression). CA-125 was significantly more likely to be raised in $\mathrm{HR}+(46.2 \%, \mathrm{p}=0.02)$ and $\mathrm{TN}$ tumors $(69.2 \%, \mathrm{p}=0.001)$ than HER2+ tumors (23.9\%). CEA was significantly more likely to be raised in HR+ $(45.8 \%)$ than either HER2+ $(22.2 \%, \mathrm{p}=0.01)$ or TN tumors $(15.4 \%, \mathrm{p}=0.007)$. There was no significant association between CA19.9 and tumor subtypes.

In the 71/193 (36.8\%) without a raised CA15.3 at diagnosis, the tumor subtype was significantly more likely to be a HR+ tumor (49.3\%), than either a HER2 + tumor $(58.3 \%)$, or a TN tumor $(21.1 \%, \mathrm{p}<0.0001$, one sample Kolmogorov-Smirnov test). In the 33/189 (17.5\%) without any raised marker at diagnosis, the tumor subtype was significantly more likely to be a HR+ tumor (45.5\%) than either a HER2+ tumor (36.4\%) or a TN tumor (18.2\%, $\mathrm{p}<0.0001$, one sample Kolmogorov-Smirnov test).

A logistic regression was performed. The group of 12/168 (7.1\%) patients with all four TMs raised at diagnosis was taken as the reference group. Those that had no raised TMs $(33 / 189,17.5 \%)$ were $6.0(95 \% \mathrm{CI}=1.13-31.7)$ times more likely to have a HER2+ tumor or a TN tumor than a HR+ tumor $(\mathrm{p}=0.04)$. Those that only had a raised CA-125 $(86 / 191,45.0 \%)$ were $6.4(95 \% \mathrm{CI}=1.05-39.3)$ times more likely to have a HER2+ tumor or a TN tumor than a HR+ tumor $(\mathrm{p}=0.04)$.

Tumor markers \& sites of metastases

The following raised TMs were significantly associated with metastases in the following sites: CA15.3 and bone metastases $(\mathrm{p}=0.03)$, CA-125 and pleural/peritoneal metastases $(\mathrm{p}<0.001)$ and CA19.9 and nodal metastases 
Table 5. Tumor markers according to sites of metastases at diagnosis; analysis by stepwise logistic regression.

\begin{tabular}{|c|c|c|c|c|c|c|c|c|c|c|}
\hline \multirow[t]{3}{*}{ Marker } & \multicolumn{10}{|c|}{ Sites of metastases } \\
\hline & \multicolumn{2}{|c|}{ Pleural/peritoneal } & \multicolumn{2}{|c|}{ Visceral } & \multicolumn{2}{|c|}{ Bone } & \multicolumn{2}{|c|}{ Nodal } & \multicolumn{2}{|c|}{ Bone only } \\
\hline & Raised (\%) & $\mathrm{OR}(\mathrm{Cl})$ & Raised (\%) & OR (Cl) & Raised (\%) & $\mathrm{OR}(\mathrm{Cl})$ & Raised (\%) & OR (Cl) & Raised (\%) & OR (Cl) \\
\hline CA15.3 & $21 / 34(61.7)^{\dagger}$ & NA & $70 / 108(64.8)^{\dagger}$ & NA & $84 / 123(68.3)^{\ddagger}$ & $2.1(1.1,4.0)$ & $53 / 86(61.6)^{\dagger}$ & NA & $25 / 38(65.8)^{\dagger}$ & NA \\
\hline CEA & $11 / 34(32.4)^{\dagger}$ & NA & $38 / 109(34.9)^{\dagger}$ & NA & $50 / 123(40.7)^{\dagger}$ & NA & $29 / 86(33.7)^{\dagger}$ & NA & $19 / 38(50.0)^{\dagger}$ & NA \\
\hline CA-125 & $26 / 34(76.5)^{\S}$ & $4.5(1.9,10.8)$ & $54 / 108(50.0)^{\dagger}$ & NA & $54 / 123(43.9)^{\dagger}$ & NA & $43 / 85(50.6)^{\dagger}$ & NA & $13 / 38(34.2)^{\dagger}$ & NA \\
\hline CA19.9 & $9 / 30(30.0)^{\dagger}$ & NA & $19 / 94(20.2)^{\dagger}$ & NA & $11 / 112(9.8)^{\dagger}$ & NA & $19 / 75(25.3)^{\mathbb{I}}$ & $2.8(1.2,6.5)$ & $2 / 34(5.9)^{\dagger}$ & NA \\
\hline \multicolumn{11}{|c|}{$\begin{array}{l}\dagger \text { Not statistically significant. } \\
\ddagger p=0.03 . \\
\$ p=0.001 . \\
\mathbb{I}_{p}=0.02 . \\
\text { NA: Not applicable; OR: Odds }\end{array}$} \\
\hline
\end{tabular}

Table 6. Individual raised tumor markers and survival.

\begin{tabular}{|c|c|c|}
\hline Tumor markers and survival & Survival marker raised & Survival marker not raised \\
\hline CA15.3 & 34.9 months & 54.6 months, $p=0.05$ \\
\hline CEA & 44.1 months & 40.1 months $^{\dagger}$ \\
\hline CA-125 & 23.1 months & 47.9 months, $p=0.007$ \\
\hline CA19.9 & 28.8 months & 42.2 months $^{\dagger}$ \\
\hline
\end{tabular}

$\dagger$ Not statistically significant.

( $p=0.02$, Fisher's exact two-tail test). See Table 5 . There was a linear association between the number of metastatic sites and the number of raised TMs $(r=0.24, p=0.002$, Pearson's test). A raised CA-125 or a raised CA19.9 was more strongly associated with the number of sites of metastatic disease than a raised CA15.3 or a raised CEA ( $\mathrm{p}=0.001, \mathrm{p}=0.03$, respectively).

\section{Tumor markers \& survival}

Median survival for all subjects was 41.7 months (range 31.1-52.3 months). The median survival for patients with HER2 + tumors (62.1 months), was not significantly different to that for patients with HR+ tumors (42.2 months), but the median survival for patients with TN tumors (10.8 months) was significantly shorter than for patients with $\mathrm{HR}+($ hazard ratio $=2.69,95 \% \mathrm{CI}=1.60-4.52, \mathrm{p}<0.0001$ ) or HER2 + tumors (hazard ratio $=10.0,95 \%$ $\mathrm{CI}=2.35-43.5, \mathrm{p}<0.0001$, Cox regression). There was no significant association between the year of diagnosis and survival ( $\mathrm{p}=0.9$, Cox regression).

With respect to individual TMs and survival, the median survival for those with a raised CA15.3 was 34.9 months and was significantly shorter than for those with a normal CA15.3 (54.6 months, p = 0.05, Kaplan-Meier and Cox regression). See Table 6. The median survival for those with a raised CA-125 was 23.1 months and was significantly shorter than for those with a normal CA-125 (47.9, p =0.006, Figure 1). There was no significant association between a raised CEA or a raised CA19.9 and survival. There was a significant inverse relation between the number of raised TMs and survival ( $\mathrm{p}=0.04$, Cox regression, Figure 2$)$. The median survival according to TM count was: no raised TM 62.1 months, one raised TM 39.0 months, two raised TMs 41.7 months, three or more raised TMs 15.5 months.

Of the 168 with results for all four TMs at diagnosis, a raised CA-125 was the most important TM with respect to survival ( $\mathrm{p}=0.03$, Cox regression) and was more important than the sites metastases and the number of metastases and was independent of tumor subtype.

\section{Multivariable analyses}

The first multivariable analysis (MVA) was for tumor subtype. Patient age, individual raised TMs, the number of raised TMs, sites of metastases and combinations of raised TMs were entered into the model. HR+ tumors were associated with a higher number of raised TMs than either HER2+ ( $p=0.02)$ or TN tumors $(p<0.0001$, multinomial logit Chi-square test) and significantly fewer sites of metastases than either HER2+ or TN tumors $(\mathrm{p}<0.0001)$. HR + tumors were also associated with bone metastases $(\mathrm{p}=0.001)$. A raised CA-125 was significantly 
CA15.3

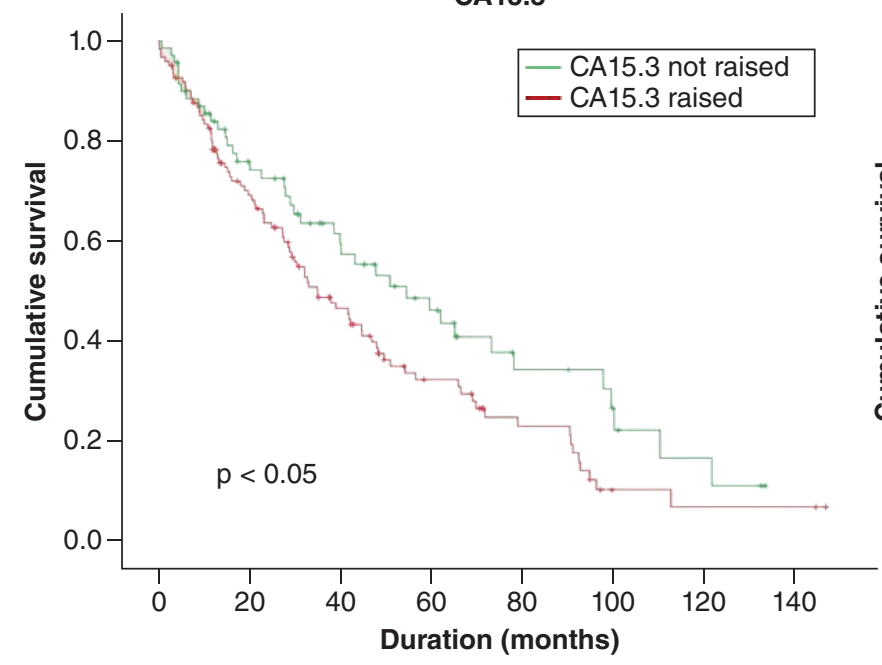

CA-125

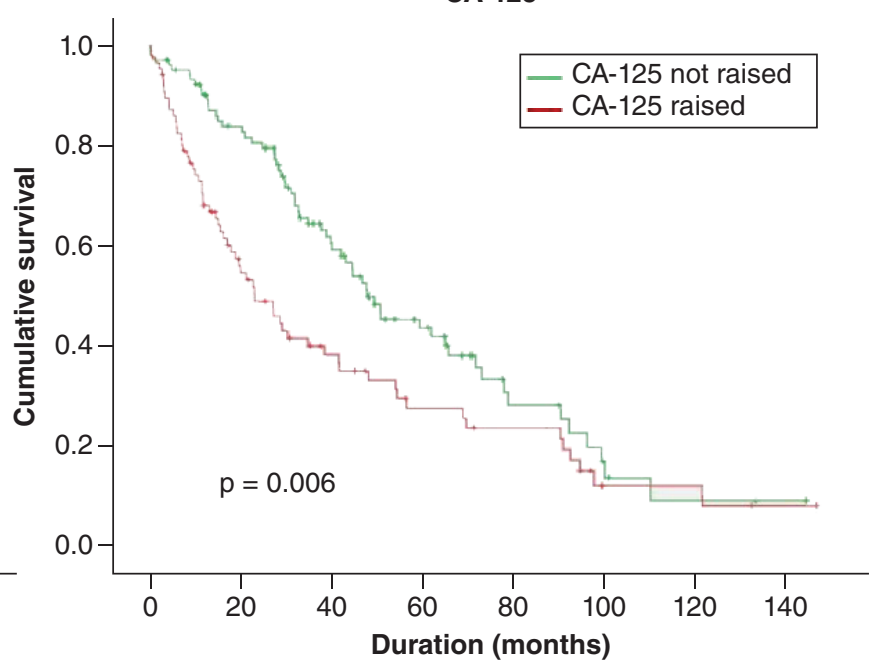

Figure 1. Survival according to whether CA15.3 and CA-125 raised or not raised.

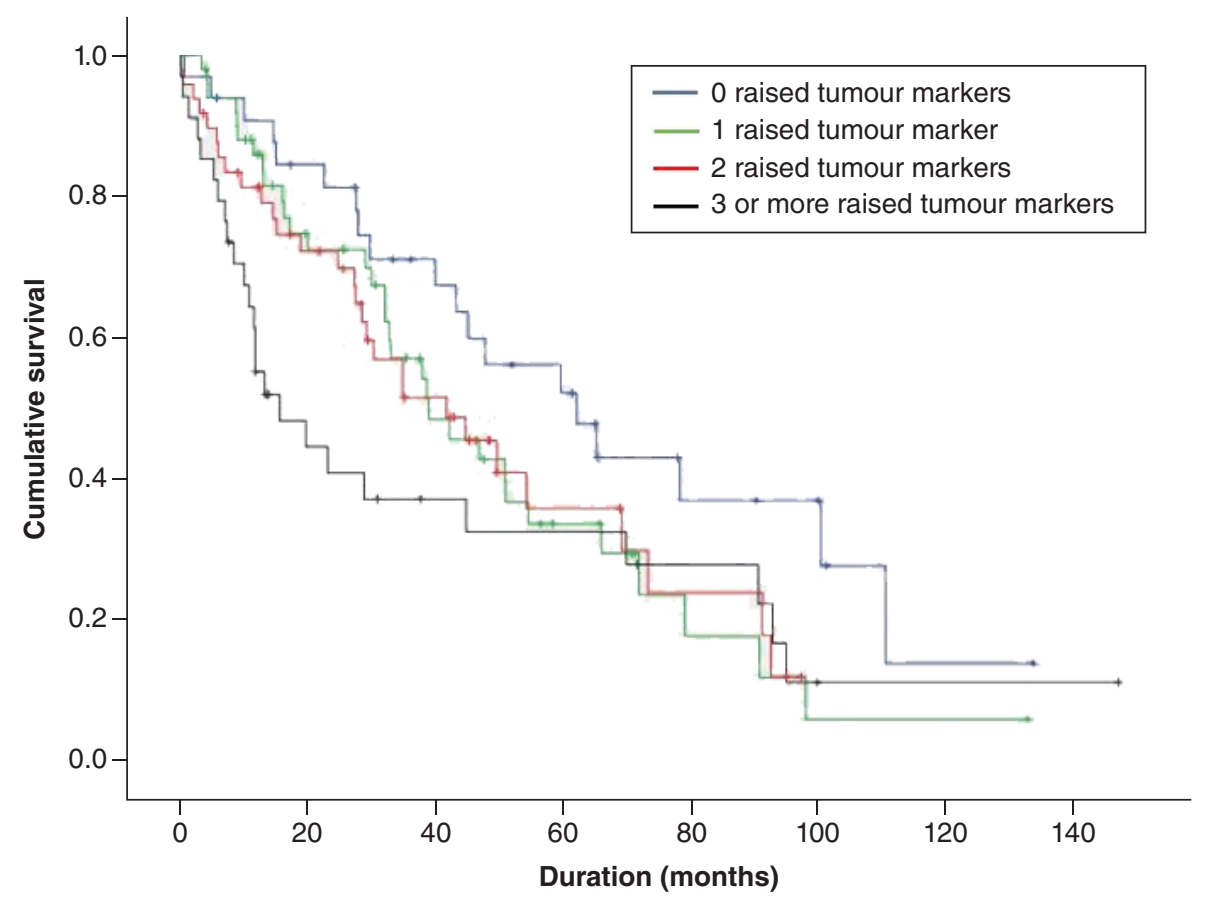

Figure 2. Survival according to the number of raised tumor markers.

more likely in TN than HR+ tumors $(\mathrm{p}=0.002)$ but not HER2+ tumors. A raised CA19.9 was significantly more likely in TN than HR+ tumors $(\mathrm{p}=0.039)$.

The second MVA was for the number of raised TMs. Patient age, tumor subtype, the sites of metastases and the number of sites of metastases were entered into the model. The number of raised TMs was significantly associated 
with the number of sites of metastases ( $\mathrm{p}<0.001$, Poisson regression, Wald Chi-square) and tumor subtype, with $\mathrm{HR}+$ tumors having a higher number of raised TMs than HER2+ tumors $(\mathrm{p}=0.001)$.

The third MVA was for survival. Patient age, tumor subtype, individual raised markers, the number of raised markers and the number of sites of metastases were entered into the model. Older age $(p=0.02)$, TN tumors $(\mathrm{p}<0.001)$ and a raised CA15.3 ( $\mathrm{p}=0.01$, Cox regression) were the most important factors associated with shorter survival.

\section{Discussion}

Most clinicians use CA15.3 or CA27.29 in the diagnosis and monitoring patients with MBC. However, in this study we used a panel of TMs in MBC, rather than CA15.3 alone. In our study, although CA15.3 was the most commonly raised marker at diagnosis, it was not raised in 71/193 (36.8\%) and never became raised in 43/193 (22.3\%). Adding other TMs to CA15.3 raised the proportion of patients with one or more raised TM at diagnosis from $122 / 193(63.2 \%)$ to $156 / 189(82.5 \%)$, clearly demonstrating the benefit of a panel of TMs in those with a normal CA15.3 at diagnosis. With respect to the other TMs, there was a significant association between the number of raised TMs and the number of sites of metastases, presumably reflecting the extent of disease. Although CEA was associated with HR+ tumors and increased the proportion of patients with a raised TM in those with a normal CA15.3 at diagnosis, it was not associated with sites of metastases or survival. CA19.9 was only elevated in a small number of subjects at diagnosis and added little information in follow-up and was not associated with survival. Therefore, we do not recommend routine CA19.9 monitoring in patients with MBC. CA-125 provided more patients with a raised TM in those with a normal CA15.3 than either CEA or CA19.9. CA-125 was also associated with shorter survival, probably due to its association with TN tumors. CA-125 was also strongly associated with the presence of pleural/peritoneal metastases. However, on the MVA for survival, CA15.3 was more important than CA-125.

A recent study examined CA15.3, CEA, CA-125 and CA19.9 in 164 patients with MBC and found that CEA had the highest sensitivity and CA-125 had the highest specificity in the diagnosis of $\mathrm{MBC}$ when using just one marker [11]. We found that adding both CEA and CA-125 increased the sensitivity of the biochemical diagnosis of MBC, similar to the findings of the study by Yerushalmi et al. [8]. Combining different TMs has also previously been shown to have merit over a single marker, both in the diagnosis and the monitoring of MBC [13].

We found a significant association between the number of raised TMs and shorter survival. This is supported by several prior studies of TMs in MBC $[8,14,15]$. However, we accept that TMs as a surrogate for prognosis is open to bias. Although our retrospective study has attempted to address certain biases with respect to survival through multivariable analysis, bias will remain. The association between the number of raised TMs and survival may simply reflect greater disease burden. [16] Our results could support this hypothesis, given that a higher number of raised TMs was associated with a higher number of sites of metastases.

Unlike early breast cancer, few previous studies have monitored several TMs during the course of the disease in patients with MBC. Our data show that if a clinician desires a TM to follow on an MBC patient, continuing to monitor CA15.3 is worthwhile even when it was normal at diagnosis, as it became raised later in 28/71 (39.4\%) of that cohort. However, from diagnosis, a normal CA15.3 took an average of 23.4 months to rise. Adding CEA, CA-125 and CA19.9 to CA15.3, increased the number of patients with a raised marker at diagnosis from 122/193 (63.2\%) to $156 / 189$ (82.5\%).

We have insufficient data to indicate whether raised TMs other than CA15.3 led to a change in treatment or prompted earlier recognition of disease progression in our patients. Nevertheless, we would argue that they probably can, given previously published data that support the utility of a raised TM in guiding treatment $[17,18]$. Even in an era of high-quality imaging, imaging may fail to indicate disease progression in those with micrometastases and eventual radiographic evidence of metastases can take up to 6 months after raised TMs are noted $[4,14]$. TMs are quick, cost-effective tests and also may provide prognostic information, as demonstrated in our study. TMs are also capable of improving diagnostic accuracy during surveillance in the case of equivocal imaging [14,18]. With respect to cost, locally each TM test costs approximately $\$ 20$, or $\$ 320$ per year for a four TM panel or $\$ 240$ per annum for a three TM panel. We believe that $\$ 60$ for a three TM panel at diagnosis is readily justifiable, given the likelihood that the panel will provide at least one raised TM that the clinician can use to monitor the patient's progress. Moreover, monitoring raised TMs may lead to a less frequent requirement for repeat imaging. However, it is more difficult to justify the costs of regular monitoring with a panel of three TMs in those without a raised TM at diagnosis $(33 / 189,17.5 \%$ in our study). Furthermore, with ongoing testing, there is greater possibility of 
discrepancy between TM test results and the results of repeat imaging and the anxiety that this may cause. The antithesis to this argument, being earlier detection and avoidance of anxiety with less frequent imaging if a raised marker is found $[4,14]$.

The second aim of our study was to explore the relationship between raised TMs and tumor subtypes and sites of metastases. We anticipated that we might find a positive association between CA-125 and pleural/peritoneal disease as this has been described previously $[19,20]$ and we did. Previous studies have also described an association between raised CA15.3 and bone metastases [21,22], which was also supported by our findings.

With respect to tumor subtype, two previous studies have reported that raised TMs occur less frequently in TN tumors than in other tumor subtypes $[8,23]$ and other studies have suggested that CEA is less likely raised to be raised in TN tumors $[8,11,24]$. We did not confirm that raised TMs occur less frequently in TN tumors but in our series, the incidence of raised TMs was significantly lower in HER2+ tumors than HR+ or TN tumors. However, we did find that the incidence of a raised CEA was significantly lower in TN tumors than in HR+ tumors.

A clinician might question the utility of TMs given the increasing use of circulating tumor cells (CTCs) and circulating tumor DNA (ctDNA). However, like TMs, CTCs and ctDNA will not be detected in all patients and it is likely that TMs will continue to be used in the routine monitoring of MBC [25-27]. In a recent study, the prognostic value of TMs in MBC was shown to be complementary to that of CTCs, with those with normal TMs and negative CTCs having the best survival [25]. In MBC, where regular monitoring of disease status is required, biomarker measurement needs to be cost effective and reliable and this has been validated for CA15.3 and CEA in the current ASCO guidelines [4]. ctDNA and CTC analysis are costly and not available routinely and it is unlikely that an oncologist will be able to access these technologies as frequently as TMs. It is more likely that, upon suspicion of disease progression, an oncologist will access their patient's ctDNA, which appears to have superior sensitivity and utility to CTCs by present technology, [28] to establish targetable mutations, returning to routine TM measurement, thereafter. In those with no raised TMs at diagnosis $(33 / 189,17.5 \%$ in our study) more frequent ctDNA monitoring may be justified.

In conclusion, our study assessed the use of a panel of TMs in a large sample of MBC patients and confirmed some of the findings of previous studies of TMs in MBC with sites of metastases (for example, CA-125 and pleural metastases), tumor subtype (for example CA-125 and TN tumors) and the association of raised TMs with shorter survival. Although in our study $95.3 \%$ of subjects had a biopsy proven diagnosis, the application of our data is particularly relevant to those suspected of clinical recurrence without the initial support of a biopsy, given this is a more common clinical scenario due to same-day TM availability and/or difficult biopsy location. We recommend measuring CA15.3, CEA and CA-125 in all new MBC patients at diagnosis to increase the sensitivity of the biochemical diagnosis of $\mathrm{MBC}$, to provide a raised TM or TMs for monitoring disease progress and to provide prognostic information. We also recommend that, in the event of a normal CA15.3 at diagnosis, the clinician should continue to monitor CA15.3, CEA and CA-125, but not CA19.9, on a regular basis in patients with MBC.

\section{Summary points}

- First paper to longitudinally assess the usefulness of an extended tumor marker panel including cancer antigen 15.3 (CA15.3), cancer antigen 125 (CA-125), cancer antigen 19.9 (CA 19.9) and carcinoembryonic antigen (CEA).

- First paper to specifically address the question of what to do in the event of a normal CA15.3 at diagnosis of metastatic breast cancer.

- Found that an extended panel of markers including CEA and CA-125 but not cancer antigen 19.9 in addition to CA15.3 provided greater diagnostic potential, particularly CA-125.

- Found that in the event of a negative CA15.3 at diagnosis, a wider panel was of benefit particularly CEA.

- Found benefit in continuing to measure CA15.3 in follow-up in the event of a normal CA15.3 at diagnosis.

- Found a correlation of number of raised tumor markers and shorter survival.

- Several tumor marker patterns were found with respect to tumor type and location of metastases that require further exploration. 
Financial \& competing interests disclosure

The authors have no relevant affiliations or financial involvement with any organization or entity with a financial interest in or financial conflict with the subject matter or materials discussed in the manuscript. This includes employment, consultancies, honoraria, stock ownership or options, expert testimony, grants or patents received or pending, or royalties.

No writing assistance was utilized in the production of this manuscript.

Ethical conduct of research

The authors state that they have obtained appropriate institutional review board approval or have followed the principles outlined in the Declaration of Helsinki for all human or animal experimental investigations. In addition, for investigations involving human subjects, informed consent has been obtained from the participants involved.

\section{Open access}

This work is licensed under the Attribution-NonCommercial-NoDerivatives 4.0 Unported License. To view a copy of this license, visit http://creativecommons.org/licenses/by-nc-nd/4.0/

\section{References}

Papers of special note have been highlighted as: • of interest

1. Early Breast Cancer Clinical Trialists' Collaborative Group (EBCTCG). Effects of chemotherapy and hormonal therapy for early breast cancer on recurrence and 15-year survival: an overview of the randomised trials. Lancet 365(9472), 1687-1717 (2005).

2. Stuart-Harris R, Dahlstrom JE, Gupta R, Zhang Y, Craft P, Shadbolt B. Recurrence in early breast cancer: analysis of data from 3,765 Australian women treated between 1997 and 2015. Breast 44, 153-159 (2019).

3. Vondeling GT, Menezes GL, Dvortsin EP et al. Burden of early, advanced and metastatic breast cancer in The Netherlands. BMC Cancer 18(1), 262 (2018).

4. Harris L, Fritsche H, Mennel R et al. American Society of Clinical Oncology 2007 update of recommendations for the use of tumor markers in breast cancer. J. Clin. Oncol. 25(33), 5287-5312 (2007).

5. Gion M, Mione R, Leona AE et al. CA27.29: a valuable marker for breast cancer management. A confirmatory multicentric study on 603 cases. Eur. J. Cancer 37(3), 355-363 (2001).

6. Duffy MJ, Shering S, Sherry F, McDermott E, O’Higgins N. CA-15.3: a prognostic marker in breast cancer. Int. J. Biol. Markers 15(4), $330-333$ (2000).

7. Ordonez C, Screaton RA, Ilantzis C, Stanners CP. Human carcinoembryonic antigen functions as an inhibitor of anoikis. Cancer Res. 60(13), 3419-3424 (2000).

8. Yerushalmi R, Tyldesley S, Kennecke $\mathrm{H}$ et al. Tumor markers in metastatic breast cancer subtypes: frequency of elevation and correlation with outcome. Ann. Oncol. 23(2), 338-345 (2011).

- Complimentary findings to this paper.

9. Berruti A, Tampellini M, Torta M, Buniva T, Gorzengo G, Dogliotti L. Prognostic value in predicting overall survival of two mucinous markers: CA 15-3 and CA 125 in breast cancer patients at first relapse of disease. Eur. J. Cancer 30A(14), 2082-2084 (1994).

10. Rump A, Morikawa Y, Tanaka M et al. Binding of ovarian cancer antigen CA125/MUC16 to mesothelin mediates cell adhesion. J. Biol. Chem. 279(10), 9190-9198 (2004).

11. Wang W, Xu X, Tian B et al. The diagnostic value of serum tumour markers CEA, CA19-9, CA125, CA15.3 and TPS in metastatic breast cancer. Clin. Chim. Acta 470, 51-55 (2017).

12. Poruk KE, Gay DZ, Brown K et al. The clinical utility of CA 19-9 in pancreatic adenocarcinoma: diagnostic and prognostic updates. Curr. Mol. Med. 13(3), 340-351 (2013).

13. Cheung KL, Graves CRL, Robertson JFR. Tumour marker measurements in the diagnosis and monitoring of breast cancer. Cancer Treat. Rev. 26(2), 91-102 (2000).

14. Duffy MJ. Serum tumor markers in breast cancer: are they of clinical value? Clin. Chem. 52(3), 345-351 (2006).

- Useful review of tumor markers in breast cancer in general.

15. Lee JS, Park S, Park JM, Cho HJ, Kim SI, Park BW. Elevated levels of serum tumor markers CA 15-3 and CEA are prognostic factors for diagnosis of metastatic breast cancers. Breast Cancer Res. Treat. 141(3), 477-484 (2013).

16. Colomer R, Ruibal A, Salvador L. Circulating tumor marker levels in advanced breast cancer correlate with the extent of metastatic disease. Cancer 64(8), 1674-1681 (1989).

17. Leonard GD, Low JA, Berman AW, Swain SM. CA 125 elevation in breast cancer: a case report and review of the literature. Breast J. 10(2), 146-149 (2004).

18. Nicolini A, Carpi A, Ferrari $\mathrm{P}$ et al. The role of tumour markers in improving the accuracy of conventional chest $\mathrm{x}$-ray and liver echography in the post-operative detection of thoracic and liver metastases from breast cancer. Br. J. Cancer 83(11), 1412-1417 (2000). 
19. Kramer S, Jager W, Lang N. CA 125 is an indicator for pleural metastases in breast cancer. Anticancer Res. 17(4B), 2967-2970 (1997).

20. Norum LF, Erikstein B, Nustad K. Elevated CA 125 in breast cancer - a sign of advanced disease. Tumor Biol. 22(4), 223-228 (2001).

21. O'Brien DP, Horgan PG, Gough DB, Skehill R, Grimes H, Given HF. CA15-3: a reliable indicator of metastatic bone disease in breast cancer patients. Ann. R. Coll. Surg. Engl. 74(1), 9-11 (1992).

22. Arslan N, Serdar M, Deveci $S$ et al. Use of CA15-3, CEA and prolactin for the primary diagnosis of breast cancer and correlation with the prognostic factors at the time of initial diagnosis. Ann. Nuc. Med. 14(5), 395-399 (2000).

23. He ZY, Li X, Chen QS et al. Elevated serum carcinoembryonic antigen and CA-15.3 levels and the risk of site-specific metastases in metastatic breast cancer. Transl. Cancer Res. 5(5), 529-537 (2016).

24. Wu SG, He ZY, Zhou J et al. Serum levels of CEA and CA15-3 in different molecular subtypes and prognostic value in Chinese breast cancer. Breast 23(1), 88-93 (2014).

25. Shiomi-Mouri Y, Kousaka J ando T et al. Clinical significance of circulating tumor cells (CTCs) with respect to optimal cut-off value and tumor markers in advanced/metastatic breast cancer. Breast Cancer 23(1), 120-127 (2016).

26. Wan JCM, Massie C, Garcia-Corbaco J et al. Liquid biopsies come of age: towards implementation of circulating tumour DNA. Nat. Rev. Cancer 17(4), 223-238 (2017).

27. Bettegowda C, Sausen M, Leary RJ et al. Detection of circulating tumor DNA in early- and late-stage human malignancies. Sci. Transl. Med. 6(224), 224ra24 (2014).

28. Dawson SJ, Tsui DWY, Murtaza M et al. Analysis of circulating tumor DNA to monitor metastatic breast cancer. N. Engl. J. Med. 368(13), 1199-1209 (2013). 